\title{
Prospects and Challenges of Afan Oromo: A Commentary
}

\author{
Tamene Keneni Walga \\ Department of Psychology, College of Social Sciences \& Humanities, Debre Berhan University, Ethiopia
}

\begin{abstract}
Afan Oromo- the language of the Oromo- is also known as Oromo. The word 'Oromo' refers to both the People of Oromo and their language. It is one of the widely spoken indigenous African languages. It is also spoken in multiple countries in Africa including Ethiopia, Kenya, Somalia, Sudan and Tanzania among others. Moreover, it is spoken as a native language, second language and lingua-franca across Ethiopia and beyond. Regardless of its scope in terms of number of speakers and geographical area it covers, Afan Oromo as a literary language is only emerging due to perpetuating unfair treatment it received from successive Ethiopian regimes. This commentary sought to examine prospects and challenges of Afan Oromo. To this end, drawing on existing literature and author's own personal observations, salient prospects and challenges of Afan Oromo have been presented and briefly discussed. Suggestions to confront the challenges foreseen have been proposed by the author where deemed necessary. The paper concludes with author's concluding remarks concerning the way forward.
\end{abstract}

Index Terms-Afan Oromo, Oromo, Oromia, Oromo people, Ethiopia

\section{INTRODUCTION}

Afan Oromo is among the ten top languages in Africa in terms of the number of native speakers. It is the most widely spoken language as a native language in Ethiopia. Moreover, it is one of the languages spoken in multiple countries in Africa, particularly East Africa. Afan Oromo is spoken in Ethiopia, Kenya, Sudan, Tanzania and Somalia among others (Wondimu, 2016). In Ethiopia, it is also spoken in several regions at different eminences. In Oromia, it is an official language; it also is a language of instruction and administration. In Amhara Regional State, it is a language of instruction and administration in Oromo Nation's Zone, one of the administrative zones in Amhara Regional State. It is also a language of instruction and administration in Harari Regional State along with Harari language. Afan Oromo is also widely spoken and used in other regions adjoining Oromia such as Afar, Benishengul-Gumuz, Somali, Gambella, and South Nations, Nationalities and Peoples Region (SNNPR). Overall, Afan Oromo is spoken as a native language, second language and lingua-franca across Ethiopia and beyond (Samuel, 2011). This paper seeks to look at the prospects and challenges of Afan Oromo drawing on literature and personal observations.

\section{HISTORY OF AFAN OROMO: AN OVERVIEW}

\section{A. Afan Oromo Writing before 1991}

Afan Oromo is one of the resilient languages in the world in general and in Africa in particular that survived colonial attack aimed at vanishing it. Its resilience is evident in its resistance to extermination in the face of the war against it by consecutive Abyssinian/Ethiopian regimes over the past 150 or so years.

Consecutive regimes of Ethiopia had done everything in their reach to exterminate the Oromo and other nations in the south and their language and culture. They used physical and biological war to eliminate the Oromo and other nations from the face of the earth and psychological war to emasculate their culture, identity and language using pretexts such as unification and nation-state formation. Haile Silassie I who used to call himself a God's elect king of kings and ruled Ethiopia for more than 50 years banned all languages spoken in Ethiopia including Afan Oromo except Amharic. Through Haile Silassie I's language rationalization policy, Amharic was imposed on all nations in Ethiopia to systematically wipe out their languages.

The situation was no different during the military junta era also known as dergue regime. Except for literacy and media, use of Afan Oromo and other languages other than Amharic for instructional and administrative purpose was prohibited. Existing literature in Afan Oromo including the Holy Book translated by Onesmos Nasib in Gee'ez script were burnt (Gamta, 2000). Looking at the series of hurdles that Tilahu Gamta had to pass through to publish a single Oromo-English Dictionary (OED) at Addis Ababa University in mid-1980 is a notable portrayal of the worst situation of Afan Oromo during the dergue regime (for full story see Gamta, 2000). What's surprising is to join higher education institutions in the country such as Addis Ababa University where medium of instruction was entirely English, students were required to earn at least ' $\mathrm{C}$ ' in Amharic in the then Ethiopian School Leaving Certification Examination(ESLCE)(Gamta,2000). Moreover, those who were able to join university were required to pass two Amharic courses-Amharic 101 and Amharic 102 to successfully make into second year (Gamta, 2000). These were 
done on purpose to at least achieve two purposes: (1) As a policy of exclusion to exclude non-Amhara or non-Amharic speakers from the sphere of higher education; and (2) as a psychological strategy to weaken and if possible to exterminate the languages of other nations.

Although Oromo literacy began in 1898(Bulcha, 1995) all endeavors by foreign scholars and Oromo intellectuals to further develop Afan Oromo writing before 1991 had been aborted by flagrant policies of successive Abyssinian/ Ethiopian regimes( for details, see Gamta, 2000 \& Wondimu, 2016). These flagrant policies include inane propagandas to belittle the other languages. For example, earlier Abyssinian/Ethiopian language and literature 'elites' had preached a lot that Afan Oromo cannot be a written language. There was also time in history when they used to tell us Afan Oromo breaks radio and hence cannot be aired on radio- it's not suited for electronic media such as radio and television.

\section{B. Afan Oromo Writing after 1991}

The history of Afan Oromo as a literary language can be traced back to $17^{\text {th }}$ century (Tesfaye, 2012). However, 1990's can be regarded as golden years in the history of written Afan Oromo for several reasons. First, it was during this time that Qubee (Latin Scripts) was adopted officially to transcribe Afan Oromo. Second, it was during this time that it became the language of instruction in primary schools (from grades 1-8) and taught as a subject in both primary and secondary schools (grades 1-12) across Oromia. Third, it is during this period that Afan Oromo became the language of administration and court in Oromia. Fourth, it was during this time that Afan Oromo started to be offered as a minor program at university. Addis Ababa University pioneered the opening of Afan Oromo as a minor program and Mekelle University followed its footstep. Fifth, this era was the time during which we read outstanding creative writings such as 'Godaanisa' by Dhaabaa Wayyeessaa, 'Gadoo' by Gaaddisaa Warquu, and many others. It was also during late 1990 that the then Ethiopian Television(ETV) and now Ethiopian Broadcasting Corporation(EBC) started Afan Oromo program(Sagantaa Afaan Oromoo) which used to air informative and entertainment programs among which 'Dhangaa' is unforgettable. The launching of Afan Oromo Program for half an hour at the Voice of America (VOA), regardless of harsh opposition from radical Ethiopianists in the diaspora, was another historical event in 1990's. Overall, 1990's was the era during which Afan Oromo showed incredible progress in all aspects.

After the millennium many universities such as Addis Ababa University, Jimma University, Haramaya University, and Wollega University among others have already started offering Bachelor and Masters Programs in Afan Oromo. The launching of the then Oromia Radio and Television and now Oromia Broadcasting Network (OBN) after the millennium is another turning point in the history of Afan Oromo though many regards it as a party mouthpiece than public media house. As of now, to the author's best knowledge, Addis Ababa University and Wollega University have doctoral $(\mathrm{PhD})$ programs in Afan Oromo and Afan Oromo Folklore and Literature. Overall, Afan Oromo has gone through a lot of hurdles and has come to this point. What will be the future of Afan Oromo? What are the prospects and challenges ahead of it? In the next sections of this commentary, I will try to highlight some prospects and challenges of Afan Oromo drawing on existing literature and my own personal observations.

\section{Prospects of AFAN Oromo}

Afan Oromo has a bright future for many reasons. In other words, there are several reasons to believe that Afan Oromo is highly likely to rise in the decades to come. In this section, I will highlight on major ones, however.

\section{A. Expansion of Schools and Increase in Students' Enrollment}

One of the successes registered over the last 25 or so years in Ethiopia in general and in Oromiya in particular is expansion of schools particularly primary schools both in rural and urban areas which in turn led to rapid growth in general enrollment rate of students. In Oromiya alone, it is estimated that about 10 million children are at school. In vast majority of these primary schools (grades 1-8), Afan Oromo is the medium of instruction. In addition, Afan Oromo is taught as a subject from grade one through grade 12 in Oromiya. Categorically, this makes the prospects of Afan Oromo very hopeful and brighter ever than before. In short, the ever increase in number of successive Qubee Generationsgenerations that studied in Afan Oromo as a medium of instruction using Qubee (Latin Script)- is one of the key circumstances that makes the future of Afan Oromo much more promising and positive.

\section{B. Digitalization of Electronic Media}

Advance in information and communication (ICT) helped and continues to help electronic media such as radio, television and others to flourish. Advances in ICT allowed digitalization of electronic media which in turn made broadcasting easy and less laborious. Satellite-based digital television and radio are superior over analogue ones in terms of quality and space and time. Afan Oromo has benefited a lot from this advance in ICT and digitalization of electronic media. For example, as a result of this advance in ICT and digitalization of electronic media, a handful of state- and privately-owned televisions and radios that broadcast in Afan Oromo have gone operational over the last few years.

Oromia Broadcasting Network (OBN), a media house owned by Oromia Regional State, broadcasts mostly in Afan Oromo through its TV, F.M and Short wave radios daily from 6:00 a.m. to 24:00 p.m. Oromia Broadcasting Service(OBS) is a privately-owned TV that broadcasts in Afan Oromo. Oromia Media Network (OMN) is a private 
media house established abroad five years ago by Oromo activists, scholars and Oromo Communities in the diaspora to voice the concerns of the Oromo and other oppressed nations. OMN has come home following the 'reform' underway and currently it broadcasts from Finfine in addition to its bases abroad. There is also another Satellite Television known as Oromiya News Network (ONN) which broadcasts in Afan Oromo from abroad. There are also some digital televisions and radios based abroad that have Afan Oromo programs. Religious TV channels that broadcast in Afan Oromo are on rise. Moa TV, TV Arara, and TV Fayina are few of these channels that are currently operational. Generally speaking, there are significant numbers of secular and religious televisions and radios that broadcast in Afan either from home or abroad and the contribution of these media to the current and prospect of Afan Oromo cannot be overemphasized.

\section{Launching of Afan Oromo Programs by International Broadcasting Corporations}

As already pointed out, Voice of America (VOA) has been broadcasting in Afan Oromo for half an hour from Monday to Friday over the past 20 or so years. A year or so ago, British Broadcasting Corporation (BBC) has launched its radio program in Afan Oromo. These are historical undertakings in the history of Afan Oromo and are very likely to positively influence the prospect of Afan Oromo. The author of this paper hopes that globally renowned media such as Aljazeera would follow the footsteps of VOA and BBC sooner or later.

\section{Burgeoning Oromo Diaspora Community}

The burgeoning Oromo diaspora communities across North America, Europe and Pacific (Australia and New Zealand) can be seen as an invaluable opportunity for the prospect of Afan Oromo. The jobs that some Oromo community associations in the diaspora are doing to promote Afan Oromo, Oromo cultural values and Oromo identity (Oromummaa) are very heartening. In addition, there are significant numbers of Afan Oromo speaking churches established in the diaspora by the Oromo communities. These things have enormous potential to positively impact the prospect of Afan Oromo in one way or the other.

\section{E. Social Media}

Social media such as Face Book, Twitter, Instagram, and many others have helped to overcome communication barriers like space and time. They allowed people to communicate from anywhere at any time. As a forum of communication and interaction, social media has great influence on all languages. There is no exception with Afan Oromo. Social media has allowed and continues to allow millions of Oromo and Oromo speakers over the world to communicate and interact at ease. When people communicate and interact, languages grow. Social media continues to advance allowing better communication and interactions. As social media advances and communication and interactions among the speakers better off, Afan Oromo is highly likely to grow. Therefore, social media can be regarded as a key factor that may positively impact the prospect of Afan Oromo.

\section{F. Growing Afan Oromo Scholarship}

Afan Oromo scholarship seems to be on the right track. All Ethiopian public universities found in Oromia have at least a bachelor's degree program in Afan Oromo. Some universities have master's program in Afan Oromo and/or Afan Oromo Folklore and Literature. As already said, Addis Ababa University and Wollega University have already launched Doctoral Programs in Afan Oromo. Overall, Afan Oromo Scholarship is moving forward and this growth can be taken as a positive factor for Afan Oromo's prospect.

\section{G. Budding Oromo Art and literature}

The role of creative arts such as movies, performing arts, creative writings and music in moving a language forward cannot be overemphasized. Although it is at its early stage, Oromo art and literature is budding. Notwithstanding the systematic marginalization of Oromo art and literature over the past 150 or so years, Oromo art particularly movies and music have shown unprecedented progress in terms of quantity over the past decades. Obviously, there is a positive correlation between art and literature and language development. Oromo creative art is expected to move forward in the years to come and to positively impact the prospect of Afan Oromo.

\section{Challenges of Afan Oromo}

Although the future of Afan Oromo is brighter than darker, there are foreseeable challenges that are likely to contain its progress. In this section, I will try to shade light on some of them.

\section{A. Poor Language Education}

Poor language education is one of the salient bottlenecks currently and in the future as well unless it is addressed as soon as possible. Language skill is a key factor in school success. If students don't have adequate language skills, they are less likely to understand and master other subjects for language is a medium through which they learn other subjects. Therefore, language education needs to be rigorous enough. Unfortunately, language education in Ethiopia in general and in Oromia in particular is poor. In Oromia, students study Afan Oromo and English as a subject from grade one through to grade 12. Amharic starts from grade five and goes on through to grade 12 as a subject. But, they master none 
of them. Failure to master a given language's basic skills (writing, reading, speaking \& listening) in twelve years can be attributed to nothing but poor language education and training. If this trend continues it is highly likely to compromise Afan Oromo's prospect.

\section{B. Lack of Pragmatic Language Police at Regional and Federal Level}

Language policy is pivotal for certain language's development and lack of it is obviously problematic. One obvious importance of language policy is that it minimizes problems that emanate from multilingualism (Zahoric \& Teshome, 2009). As Zahoric and Teshome (2009) rightly note, making language policy requires authority and thus it is the mandate of governments in charge. Lamentably, we have no explicit and pragmatic language policy at regional and federal levels and this lack of workable policy unquestionably harnesses the progress of Afan Oromo and other languages spoken in this country.

There are various models of language policy. Referring to Patten (2001), Zahoric and Teshome (2009) outline four models of language policy: disestablishment, official multilingualism, language rationalization and language maintenance. The disestablishment model also known as public disengagement model entails absence of publicly recognized official language or languages (Zahoric \& Teshome, 2009).The multilingualism model calls for equal recognition of various languages (Zahoric \& Teshome, 2009). The language rationalization model involves giving undue privileges to the 'public language' by either repudiating or restricting the recognition and acceptance of other languages (Zahoric \& Teshome, 2009). The language maintenance model "aims to make all languages equally successful" (Zahoric \& Teshome, 2009, p. 81).

Language policies during imperial and military regimes had clearly been language rationalization. During these regimes Amharic was unduly privileged at the expense of other languages spoken in the country. In line with this assertion, Fiseha (n.d.) states that "The language policy of Ethiopia during the imperial and the military regimes prioritized Amharic as the state's sole official language and discriminated against all other Ethiopian languages" (p.1).

Compared to previous regimes' language policy, the language policy of Ethiopian Peoples' Revolutionary Democratic Front (EPRDF)-the party in power since 1991- can be said liberal based on Article 5 of the Constitution of the Federal Democratic Republic of Ethiopia (FDRE). Article 5(1) of the Constitution reads as "All Ethiopian languages shall enjoy equal state recognition". At the same time, the Constitution, in its article 5(2), recognizes Amharic as a sole federal working language, not as an official language or as a national language, though the meaning of the phrase 'working language' is not clear and controversial. Article 5(3) stipulates that "Members of the federation may by law determine their respective working language". In principle, the Constitution of FDRE ascribes equal recognition and status to all languages spoken in Ethiopia as stipulated in article 5(1) of the FDRE's Constitution which states that "All Ethiopian languages shall enjoy equal state recognition". But, in practice this is not the case. In this regard, I concur with Fiseha's claim that "...Amharic still enjoys paramount predominance because it is recognized as the sole official working language of the Federal Government in the current Constitution” (p.1). In other words, in practice FDRE's language policy is the continuation of previous regimes' policy.

A closer look at these articles of the constitution reveals that the foundation of language policy laid in the Constitution vacillates between two language policy models-disestablishment and multilingualism. On the one hand, there is neither clearly articulated "official language" nor a "national language". This is closer to the disestablishment model. On the other hand, at least in principle it gives equal recognition to all languages spoken in Ethiopia. This is obviously a multilingual model. Another problem with EPRDF's language policy is what is stipulated in the constitution is not translated into clear and actionable language policy. Constitution is not a policy on itself; rather, it paves the way for a policy and therefore it should have been supported by clear language policy.

Of the four models highlighted here, in my opinion, the multilingual and language maintenance models fit well to Ethiopia's situation. In a multinational and multilingual country like Ethiopia, favoring one language over the others either explicitly or tacitly is perilous. As Fiseha puts rightly a grip to Amharic as Federal Government's sole official working language is likely to exacerbate conflicts that emanate from the differences over the use of language. Moreover, it can be a serious bottleneck to the inclusive governance and democracy desired in the country. In fact, this sort of language policy is not compatible with the current government arrangement for federalism without multiculturalism and multilingualism is incomplete and incompetent. Therefore, an official multilingualism at federal level is not a choice but a must.

\section{Government's Unyieldingness to People's Demand}

Closely related to the lack of appropriate language policy both at federal and regional levels is unyieldingness of the government to people's demand about making Afan Oromo an additional official working language of the Federal Government. This quest has been one of the core issues that persisted during the Oromo Protest in last five or so years but has fallen on deaf ears on part of the government. Making Afan Oromo federal government's additional official working language benefits not only the language and its speakers but also the country. Making Afan Oromo federal government's official working language along Amharic is undoubtedly a key factor in ensuring inclusive governance and government, democracy and national unity apart from its economic and socio-cultural benefits. It is also of paramount importance to bring about political stability in the country and beyond because doing so slows down Oromo People's deep-seated resentment over past and present linguistic and socio-cultural unfairness. Therefore, the 
government needs to see the demand positively and respond to it positively in time. The federal government of Ethiopia needs to learn from countries that adopted official multilingual language policy such as Nigeria, South Africa, and Switzerland among others. Nigeria, for example, adopted English as an official language and other three widely spoken languages as national languages. South Africa has twelve official languages including Sign Language. The Swiss Federation which is considered as the best example of official multilingualism recognizes four languages (German, French, Italian, and Romantsch) as the official and national language of the Switzerland (Fiseha, n.d.).

\section{Lack of Regulation}

There are numerous practical challenges related to writing Afan Oromo that are likely to hold back its progress and prospect which can be addressed through rigorous regulation. Sadly, we don't have this in place. Afan Oromo as a literary language is only emerging. As an emerging language, it requires special regulation and safeguarding. The duty and responsibility to regulate and safeguard this emerging language largely rests upon the Oromo People and the Government of Oromia. Anyhow, writing errors in Afan Oromo written expressions have become conventional everywhere (in text books, newspapers, notices, official letters, billboards, social media posts, etc.). In his work entitled "Common Mistakes Committed in Using the Oromo Writing System: The status of texts of billboards in Adama Town", Samuel, L. Ebba(2011) points out the seriousness of the problem and nicely details when, where, and how the errors are committed( for detail see Samuel, 2011).

What is saddening is these errors in writing are committed by government offices naturally meant to develop Afan Oromo. For example, if you go to Oromia's department of education, their billboard and letter head read as "Biiroo Barnoota Oromiyaa". This is not a correct expression. It should have been written as "Biiroo Barnootaa Oromiyaa". The same is true of Oromia's department of transport. Their billboard and letter head read as "Biiroo Geejjiba Oromiyaa". But, the correct expression is "Biiroo Geejjibaa Oromiyaa". The problem is even much more serious in Oromia's Colleges of Teachers' Education. Their billboards and letter heads are wrongly spelled. For example, Nekemte College of Teachers' Education is spelt as "Kolleejjii Barnoota Barsiisota Naqamtee" which doesn't convey any message. The phrase "barnoota barsiisota" is meaningless. To be meaningful, it should have been written as "barnoota barsiisotaa" which means teachers' education. When written correctly, it goes like this: "Kolleejjii Barnoota Barsiisotaa Naqamtee". What is disgusting is TV anchors and news readers do read what is wrongly written as it is rather than correcting it. Overall, public agencies such Education Department of Oromia and Colleges of Teachers' Education under its leadership and OBN that are supposed to move this emerging language ahead are doing the opposite. Therefore, serious regulation is needed. The author suggests an independent public-funded agency that is mandated to develop Afan Oromo through aggressive regulation.

\section{E. Absence of Print Media in Afan Oromo}

Print media has an irreplaceable role in a given language's development and prospect and lack of it is an obvious risk. Afan Oromo print media (such as newspapers, magazines, newsletters ... etc.) is lacking. There are three state-owned newspapers whose circulation is confined to government offices and whose annual distribution is not encouraging (Kemal, 2014). Private newspaper in Afan Oromo is totally absent. Magazines are entirely lacking and if any intermittent. Although print media is challenged by the advent of electronic newspapers and/or social media globally and in Ethiopia as well, the situation of Afan Oromo print media is dismaying.

Kemal (2014) has aptly pointed out that Afan Oromo print media is bounded by problems such as lack of professionalism, absence of reading culture, printing cost and circulation problems. However, what Kemal (2014) has observed as bounding factors are only situational or immediate cause for absence of Afan Oromo print media. The fundamental and underpinning cause that bounded print media in Afan Oromo revolves around politico-historical factors inherited from previous regimes. For example, Afan Oromo and other languages have been discriminated against in previous regimes and this has continued subtly and systematically under the current regime. It seems that language rationalization is still at work. Afan Oromo should have been one of the official working languages of the Federal Government of Ethiopia. A government that speaks a single language doesn't qualify to be called a federal government. In my opinion, one of the things that make a government a federal government is multiculturalism and multilingualism.

\section{F. Lack of Quality Publications in Afan Oromo}

In terms of quantity, publication in Afan Oromo is encouraging. Particularly, publications that revolve around creative writings such poetry and short novels and Oromo history and politics come out frequently. But, most of these publications lack quality both in terms of substance and mechanics. They are not well thought, proofread and edited. To be frank, vast majority of these publications do not read well and therefore are not sold. In fact, I don't want to say much on this matter for it needs extensive research. However, it must be underscored that quality of what is authored is a serious challenge that Afan Oromo is facing currently and likely to face in the future if it is not addressed institutionally. The author suggests that a language academy that empowers the burgeoning novice Afan Oromo writers and authors needs to be put in place to curb this problem.

G. Lack of Journals in Afan Oromo 
Journals are magazines or periodicals published by specialists or professional bodies and networks or universities for their members and for interested wider audiences as well. In short, journals are outlets through which research outcomes, scientific inventions and innovations and other scholarly products are disseminated to potential users and consumers. Journals play unparalleled part in a moving a language ahead and obviously lack of it is a menace. Each year, students in Afan Oromo programs (bachelor, master's, and doctoral) and scholars in the field produce hundreds of scholarly works in Afan Oromo. But, since there is no single journal that publishes works done in Afan Oromo as Disasa(2013) has unveiled, these works fester in university library shelves and are finally binned into dustbins. Lack of journals and other platforms to publicize scholarly works done in Afan Oromo is one of the challenges that jeopardize Afan Oromo's progress in one way or the other.

\section{H. Attitudinal Barriers}

Negative attitude toward native/domestic languages is common in Africa and is exacerbated by globalization. There is a clear tendency among the young generation to prefer foreign languages particularly English over native/domestic languages. This is can be inferred from their daily conversations and interactions. The same thing is also reflected among parents and the larger public. Disasa (2013) has shown that students' interest to join Afan Oromo programs at Jimma University has declined overtime. Based on his findings Disasa (2013) has come to the conclusion that "lack of interest" and "negative attitude toward the language" among students are among the major challenges of Afan Oromo programs at higher education institutions in Ethiopia running Afan Oromo programs. I concur with Disasa (2013) that these are realistic challenges that need to be tackled. But, I am of the opinion that this is not unique to Afan Oromo; rather, it pertains to all domestic languages spoken in Ethiopia and even in Africa. In author's opinion, this issue can be addressed through indigenization of education from pre-primary through to university.

\section{CONCLUDing REMARKS}

This commentary set out to examine prospects and challenges of Afan Oromo. To this end, drawing on existing literature and own personal observations, the author explored and highlighted major prospects and challenges of the language in view of past and present realities. Accordingly, having presented a bird's view of Afan Oromo before and after 1991, the author has outlined and briefly discussed prospects and challenges of Afan Oromo one after other. The author does not believe that the list and discussion of the prospects and challenges is exhaustive and hence calls for intensive and extensive research into the issue.

Notwithstanding the challenges raised here and other many challenges that bound the language, the author foresees brighter future of Afan Oromo. However, one thing that must be noted here is that the war against Afan Oromo that had begun 150 or so years ago is not over. Opponents of multiculturalism and multilingualism have continued and will continue to do whatever in their reach explicitly and implicitly to revert back Afan Oromo. This has been signaled in past years. For example, the gimmick move to reshuffle Qubee Afan Oromoo by the Federal Ministry of Education (FMoE) using quality of education as pretext and collaborative "scholars" as Trojan horse is good evidence to support my assertion. In connection to this, it is also wise to be cautious and hyper vigilant of the "Education Roadmap" underway. In the past three or so years, we have been witnessing 'gentle' media campaigns to console and convince the Oromo to resort to Ethiopic alphabet to transcribe Afan Oromo. In author's opinion, this is a new version of old project aimed at taking Afan Oromo back where it has been 30 years ago. The Oromo People had been paying unprecedented sacrifices to preserve their identity, culture and language over a century and half. Now, they are obliged to preserve and sustain what they had been paying heavy price for. Afan Oromo should be federal government's working language on equal plane with Amharic as soon as possible. In author's opinion, making Afan Oromo a federal working language has nothing to do with the constitution and even the constitution doesn't forbid doing so. Rather, it is a matter of political willingness and determination. Actionable language policies need to be put in place at federal and regional levels. Finally, the author underscores the necessity for crafting a strong regulating body and language academy at Oromia level to foster the use and development of Afan Oromo.

\section{REFERENCES}

[1] Bulcha, M. (1995). Onesimos Nasib's Pioneering Contributions to Oromo Writing. Nordic Journal of African Studies, 4(1), 3659.

[2] Disasa, M.L. (2013). The Status of Afan Oromo as Indigenous Language Program in Ethiopian Higher Education Institutions: An Exploratory Study of Three Selected Universities. International Journal of Science and Research (IJSR), 2319-7064.

[3] FDRE (1994). Constitution of the federal democratic republic of Ethiopia, Negarit Gazeta, 1 (1), 1-38.

[4] Fiseha, H.G. (n.d.). Choosing a Working Language in Multiethnic Nations: Rethinking Ethiopia’s Working Language Policy. Unpublished Master's Thesis.

[5] Gamta, T. (2000). The politicization of my Oromo-English Dictionary: The writer's reflection. The Journal of Oromo Studies, $4(1 \& 2), 1-17$.

[6] Patten, A. (2001). Political approach and language policy. Political Approach, 29(5), 691-715.

[7] Samuel, L.E. (2011). Common mistakes committed in using the Oromo Writing system: The status of texts of billboard in Adama Town. Saarbrucken: LAP LAMBERT Academic Publishing 
[8] Wondimu, T. (2016). The Development of Written Afan Oromo and the Appropriateness of Qubee, Latin Script, for Afan Oromo Writing. Historical Research Letter (online), 8, 8-14.

[9] Zahoric, J. \& Teshome, W. (2009). Debating Language Policy in Ethiopia. Asian and African Studies, 18(1), 80-102.

Tamene Keneni Walga obtained a BA (educational psychology), an MA (developmental psychology) and a PhD (applied developmental psychology) from Addis Ababa University in 2003, 2008 and 2016, respectively. He also obtained another MA (applied social psychology) from National Research University aka Higher School of Economics (HSE), Moscow, Russia. Tamene is an interdisciplinary teacher, researcher and practitioner. 